\title{
Transabdominal Functional Magnetic Stimulation for the Treatment of Constipation in Brain-Injured Patients: A Randomized Controlled Trial
}

\author{
Young-Cheol Yun, $\mathrm{MD}^{1}$, Yong-Soon Yoon, $\mathrm{MD}, \mathrm{PhD}^{1,2}$, Eun-Sil Kim, $\mathrm{MD}^{1}$, \\ Young-Jae Lee, $\mathrm{MD}^{3}$, Jin-Gyeong Lee, $\mathrm{MD}^{1}$, Won-Jae Jo, $\mathrm{MD}^{1}$, Kwang Jae Lee, MD, $\mathrm{PhD}^{1,2}$
} ${ }^{1}$ Department of Physical Medicine and Rehabilitation, Presbyterian Medical Center, Jeonju; ${ }^{2}$ Medical Device Clinical Trial Center,
Presbyterian Medical Center, Jeonju; ${ }^{3}$ Department of Internal Medicine, Presbyterian Medical Center, Jeonju, Korea

Objective To investigate the effects of the transabdominal functional magnetic stimulation (A-FMS) for constipation in stroke or brain-injured patients.

Methods Twenty-four brain-injured patients (11 males and 13 females; median age, 65 years; 22 cases of stroke and 2 cases of traumatic brain injury) with constipation, who were admitted to the rehabilitation department, were enrolled and randomly divided into magnetic stimulation (MS) group and sham stimulation (Sham) group. Several parameters related with constipation such as total and segmental colon transit time (CTT), defecation frequency, and Bristol Stool Scale (BSS) before and after 2 weeks of A-FMS (5 times per week, total 10 times of A-FMS) were evaluated. The Korean version of the Modified Barthel Index (K-MBI) was also evaluated.

Results A significant decrease in segmental CTT in the left colon $(-8.2 \pm 3.9$ vs. $4.1 \pm 2.5$ hours; $\mathrm{p}<0.05$ by paired sample t-test) and a significant increase in the frequency of defecation ( $1.5 \pm 0.2 \mathrm{vs} 0.7 \pm 0.3 ; \mathrm{p}<0.05$ by paired sample t-test) were observed in the MS group compared with the Sham group. Stool hardness became significantly softer in the MS group compared with the Sham group (2.3-3.5 in the MS and 2.6-3.1 in the Sham; $<<0.05$ by chi-square test) as evaluated by BSS. No difference in the K-MBI was observed between the two groups.

Conclusion The present study suggests that A-FMS can be an additional therapeutic tool for managing constipation in brain-injured patients with abnormal bowel movement, defecation frequency, and stool hardness.

Keywords Brain injuries, Stroke, Traumatic brain injuries, Constipation, Magnetic stimulation

Received August 23, 2018; Accepted October 1, 2018

Corresponding author: Kwang Jae Lee

Department of Physical Medicine and Rehabilitation and Medical Device Clinical Trial Center, Presbyterian Medical Center, 365 Seowon-ro, Wansangu, Jeonju 54987, Korea. Tel: +82-63-230-1460, Fax: +82-63-230-8680, E-mail: rytt@naver.com

ORCID: Young-Cheol Yun (http://orcid.org/0000-0003-4970-1767); Yong-Soon Yoon (http://orcid.org/0000-0003-4709-3964); Eun-Sil Kim (http:// orcid.org/0000-0003-1996-4451); Young-Jae Lee (http://orcid.org/0000-0002-3933-7343); Jin-Gyeong Lee (http://orcid.org/0000-0002-2642-7526); Won-Jae Jo (http://orcid.org/0000-0002-7124-7153); Kwang Jae Lee (http://orcid.org/0000-0001-7148-3765).

(c) This is an open-access article distributed under the terms of the Creative Commons Attribution Non-Commercial License (http://creativecommons.org/ licenses/by-nc/4.0) which permits unrestricted noncommercial use, distribution, and reproduction in any medium, provided the original work is properly cited. Copyright ( 2019 by Korean Academy of Rehabilitation Medicine 


\section{INTRODUCTION}

Defecation is a basic physiological function in all people. In general, hard fecal consistency, decreased evacuation frequency, and sensation of incomplete evacuation are observed in $2 \%-27 \%$ of the general population $[1,2]$. In patients with stroke or traumatic brain injury, various factors of the brain-gut axis between neurological and gastrointestinal systems are impaired $[3,4]$ of which constipation and fecal incontinence are the most common. In a systematic review, the incidence of constipation was noted as follows: $51 \%-66 \%$ in stroke patients, $45 \%$ in the acute stage of stroke, $48 \%$ during rehabilitation period [5], and $79.4 \%$ in stroke patients admitted to rehabilitation wards [6]. Constipation has a negative impact on social functioning and quality of life, leading to increased hospitalization, poor neurological outcome, increased incidence of complications, and death [7]. Currently, treatments for constipation after brain injury include dietary control, abdominal massage, bio-feedback, laxatives, enemas, and prokinetic agents as well as limiting drugs that lead to constipation [7]. Non-invasive functional magnetic stimulation (FMS) in combination with the abovementioned therapies promote bowel movement [8], and the positive effect of non-invasive electrical or magnetic stimulation on constipation has previously been reported [9-12]. Earlier, patients with spinal cord injury underwent either direct abdominal stimulation from the anterior abdomen (transabdominal) or via sacral FMS of the pelvic nerve at the posterior S2-4 [12]. Recently, transabdominal FMS (A-FMS) has been reported to reduce colon transit time (CTT), increase bowel movements, and reduce stool hardness in conditions of chronic constipation in stroke patients [13]. However, in the reported study, the comparison was made under stimulation and absence of stimulation in the same stroke patients and the number of subjects was limited to 12. Apparently, the present study was designed as a randomized controlled study to investigate the effect of A-FMS on constipation-movement of bowel, defecation frequency as well as fecal consistency-in brain-injured patients.

\section{MATERIALS AND METHODS}

\section{Subjects}

The brain-injured patients exhibiting no improvement in constipation by medication were admitted to the Department of Rehabilitation Medicine at Presbyterian Medical Center from July 2013 to March 2017. Thirtyone patients participated in the study but a total of 24 participants were enrolled. The subjects were included in the study based on the following inclusion criteria: (1) male/female adults aged 20 years or older diagnosed with stroke or traumatic brain injury on computerized tomography or magnetic resonance images and who had passed at least 2 weeks after stroke onset, (2) presence of stable vital signs, (3) those who consumed diet per oral or by Levin tube or by gastrostomy, and (4) those who met the criteria of functional constipation. Functional constipation was determined based on the Rome III criteria [14] (Table 1). The exclusion criteria included: (1) history of abdominal surgery, (2) past or current diagnosis of hypothyroidism, (3) small or large bowel problems-dysplasia of the anus, irritable bowel syndrome, and congenital megacolon, and (4) pregnant women.

The study was deemed appropriate and approved by the Institutional Review Board of Presbyterian Medical Center (No. 2013-01-04). The study was performed in accordance with the Declaration of Helsinki, and all the enrolled patients submitted a written informed consent

Table 1. Rome III diagnostic criteria of functional constipation

1. Must include two or more of the following

- Straining during at least $25 \%$ of defecations

- Lumpy or hard stools in at least $25 \%$ of defecations

- The sensation of incomplete evacuation for at least $25 \%$ of defecations

- The sensation of anorectal obstruction/blockage for at least $25 \%$ of defecations

- Manual maneuvers to facilitate at least $25 \%$ of defecations (e.g., digital evacuation, support of the pelvic floor)

- Fewer than three defecations per week

2. Rare presence of loose stool without the use of laxatives

3. Insufficient criteria for irritable bowel syndrome 
for participation.

\section{Clinical evaluation}

Subjects were randomly assigned to either magnetic stimulation (MS) or sham stimulation (Sham) groups using a permuted block randomization. They continued taking the laxatives as they had been taking prior to enrollment and maintained their regimen throughout the study. During the first week, the CTT, the frequency of defecation per week, stool hardness, and the Korean version of the Modified Barthel Index (K-MBI) were evaluated. Same variables were re-evaluated during the 4 th week after 2 weeks of A-FMS application. CTT was administered in capsule form (Kolomark, MITech, Pyeongtaek, Korea) containing 20 radiopacity markers at 9 a.m. for 3 consecutive days. Patients who were on tubefeeding had capsules directly inserted into the stomach via a gastroendoscope by a gastroenterologist. On the 4th day, supine simple abdomen radiography was taken and the radiograph was subdivided the colon into three segments: right colon, left colon, and rectosigmoid colon. The number of radiopacity markers present in each segment was counted and multiplied by 1.2 to calculate CTT (segmental CTT) and the total CTT was calculated by summation of its entirety [15] (Fig. 1). The frequency of defecation (times/week) was recorded. The stool hardness was classified into 1 through 7 types according to the shape and consistency using the Bristol Stool Scale (BSS) (Table 2) and the different types of stool hardness were converted into points. Investigators were aware of the group of each patient and magnetic stimulation was done by the therapists in the stimulation room, but the K-MBI, BSS, and frequency of defecation were evaluated blindly by each resident in-charge of the patients.

\section{Transabdominal FMS application}

At the 2nd week juncture, a circular coil magnetic stimulator (BioCon-1000Pro; Mcube Technology, Seoul, Korea) was applied on the segment in which radiopacity markers were retained the most as per supine simple abdomen radiograph on the 4 th day of the first week. This stimulator generates a rapidly changing magnetic field, producing a brief burst (pulse width $370 \mu \mathrm{s}$ ) of very high current with a 1.5 Tesla $(\mathrm{T})$ that is equivalent to 15,000 gauss (G), sufficient to stimulate the peripheral nerve and the intensity of the secondarily produced electrical field in nervous tissue is related to the rapidity of the change in magnetic field strength [16]. We chose $1.5 \mathrm{~T}$ intensity according to the previous reference study [12] to stimu-

Table 2. Bristol Stool scale

Type 1. Separate hard lumps, like nuts

Type 2. Sausage-shaped but lumpy

Type 3. Like a sausage or snake but with cracks on its surface

Type 4. Like a sausage or snake, smooth and soft

Type 5. Soft blobs with clear-cut edges

Type 6. Fluffy pieces with ragged edges, a mushy stool Type 7. Watery with no solid pieces
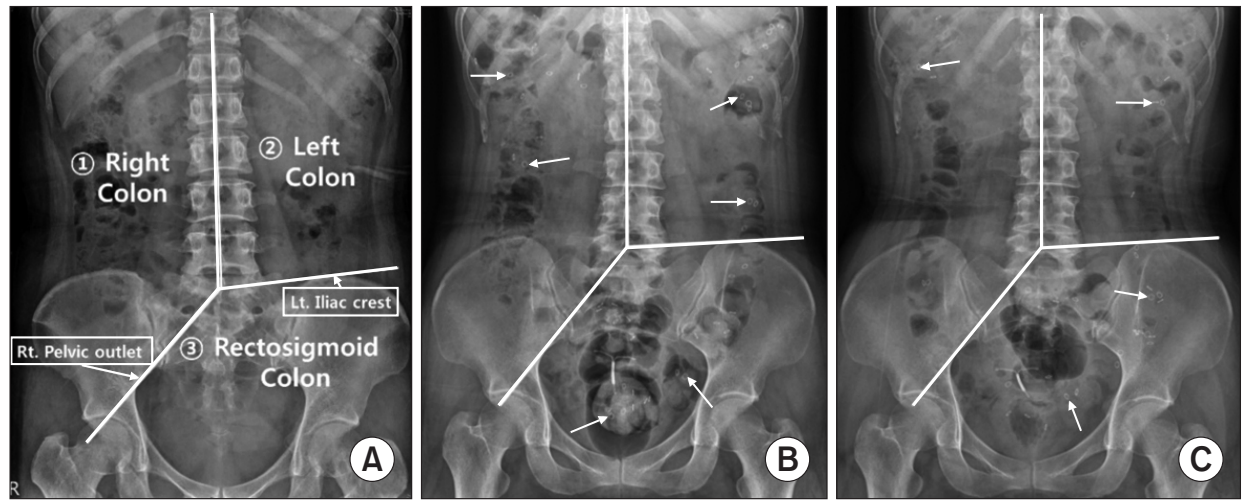

Fig. 1. Simple abdomen radiographs show (A) three segments of the colon (right, left, and rectosigmoid colon) and distribution of radiopacity markers (arrows), and (B) before and (C) after 2 weeks of transabdominal functional magnetic stimulation. Three segments were divided with imaginary lines starting from the spinous process of a 5 th lumbar vertebra to the upper spinous processes, to passing the right pelvic outlet, and to passing the left iliac crest. To calculate the colon transit time, the number of remaining radiopacity markers were counted and multiplied by 1.2 [15]. 
late mainly the pelvic splanchnic nerve and to stimulate the abdominal muscles for increasing the abdominal pressure. The secondarily induced electrical strength was $10 \mathrm{~V}$ at $1 \mathrm{~cm}$ and $3 \mathrm{~V}$ at $5 \mathrm{~cm}$ from the stimulator head. The patients were treated with magnetic stimulation in a comfortable supine position (Fig. 2). A-FMS was administered for 20 minutes daily (3-second stimulation followed by 6 -second rest) with $40 \mathrm{~Hz}$ frequency and intensity of $1.5 \mathrm{~T}$ (maximal intensity) for five times weekly for 2 weeks. In the Sham group (Fig. 2A), the intensity of $0.5 \mathrm{~T}$ (30\% of maximal intensity) was applied at a distance $5 \mathrm{~cm}$ away from the abdomen to stimulate the abdomen while using the same criteria applied for other parameters in the MS group (Fig. 2B). The patient's condition and the occurrence of any adverse events were monitored throughout the study period.

\section{Statistical analysis}

Data analysis was done by R language v3.3.3 (R Foundation for Statistical Computing, Vienna, Austria). We used Student t-test for total and segmental CTT and KMBI, Welch test and Fisher exact test for analyzing the frequency of defecation and chi-square test for assessment of fecal consistency to compare the differences in changes between the MS group and the Sham group. The level was considered significant when the p-value was less than 0.05 .

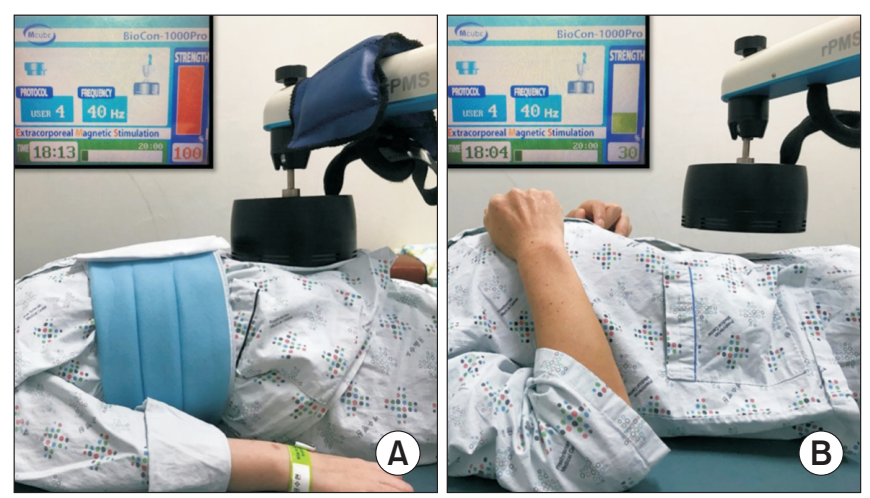

Fig. 2. Transabdominal functional magnetic stimulation is applied in an experimental patient (A) and sham stimulation in control patient (B). In (B), note that the magnetic head is $5 \mathrm{~cm}$ apart from the patient's belly and the stimulation intensity is $30 \%$ of the maximal intensity $(1.5 \mathrm{~T})$ so that the patient hears stimulation sound but does not feel the magnetic stimulation.

\section{RESULTS}

\section{General characteristics}

Of the 31 patients, 7 were dropped from the study due to discharge or transfer, resulting in a total of 24 participants (12 in the MS group and 12 in the Sham group). The mean age of the patients in the MS group was $59.7 \pm 10.9$ years (6 males and 6 females; 6 cerebral infarction and 6 hemorrhage; disease duration, $10.1 \pm 19.7$ months) and the mean age in the Sham group was $69.2 \pm 12.3$ years ( 5 males and 7 females; 5 cerebral infarction, 5 spontaneous intracranial hemorrhage, and 2 traumatic brain injury; disease duration, $16 \pm 34$ months) (Table 3). There was no difference in parameters between the two groups. The capsules containing the radiopacity markers were inserted into the stomach by a gastroendoscope in 1 patient in the MS group and in 1 patient in the Sham group while the remaining participants the route of administration was oral.

\section{Changes in CTT}

In the MS group, A-FMS was applied to the right colon segment in 6 patients, the left colon segment in 4 patients, and the rectosigmoid colon segment in 2 patients. In the Sham group, A-FMS was applied to the right colon segment in 6 patients, the left colon segment in 1 patient,

Table 3. Demographics and general characteristics of the patients

\begin{tabular}{lccc}
\hline & $\begin{array}{c}\text { Sham group } \\
(\mathbf{n}=12)\end{array}$ & $\begin{array}{c}\text { MS group } \\
(\mathbf{n}=12)\end{array}$ & p-value \\
\hline Age (yr) & $69.2 \pm 12.3$ & $59.7 \pm 10.9$ & 0.059 \\
Sex & & & 0.682 \\
$\quad$ Male & 5 & 6 & \\
\hline Female & 7 & 6 & \\
Age (yr) & $69.2 \pm 12.3$ & $59.7 \pm 10.9$ & 0.059 \\
Weight (kg) & $61.8 \pm 10.8$ & $60.7 \pm 8.4$ & 0.549 \\
Type of injury & & & 0.523 \\
CI & 5 & 6 & \\
\hline S-ICH & 5 & 6 & \\
TBI & 2 & 0 & \\
Onset (mo) & $16.0 \pm 34.0$ & $10.1 \pm 19.7$ & 0.977 \\
\hline
\end{tabular}

Values are presented as mean \pm standard deviation.

MS, magnetic stimulation; CI, cerebral infarction; S-ICH, spontaneous intracranial hemorrhage; TBI, traumatic brain injury. 
and the rectosigmoid colon segment in 5 patients. Total CTT decreased from $54 \pm 4.9$ hours before treatment to $43.2 \pm 5.3$ hours after treatment in the MS group, and increased from $40.4 \pm 6.1$ hours before treatment to $46.3 \pm 5.7$ hours after treatment in the Sham group (Fig. 3A). In the MS group, the segmental CTTs in the right colon and the rectosigmoid colon were $24 \pm 4.1$ and $7.4 \pm 2.5$ hours (before treatment) and 18.9 \pm 3.6 and 9.9 \pm 2.7 hours (after treatment), respectively, and in the Sham group, 17.5 \pm 5.6 and $14.4 \pm 3.5$ hours (before treatment) and $15.4 \pm 4.3$ and 18.3 \pm 4.4 hours (after treatment), respectively (Fig. 3B, $3 \mathrm{D})$. There was no statistical significance between the two groups in total and segmental CTT of the right and rectosigmoid colon. Only the segmental CTT in the left colon was statistically significant between the changes in the MS group (from $22.6 \pm 3.8$ to $14.4 \pm 3.1$ hours) and the changes in the Sham group (from $8.5 \pm 1.7$ to $12.6 \pm 2.2$ hours) ( $p=0.014$, Student t-test) (Fig. 3C).

(A)

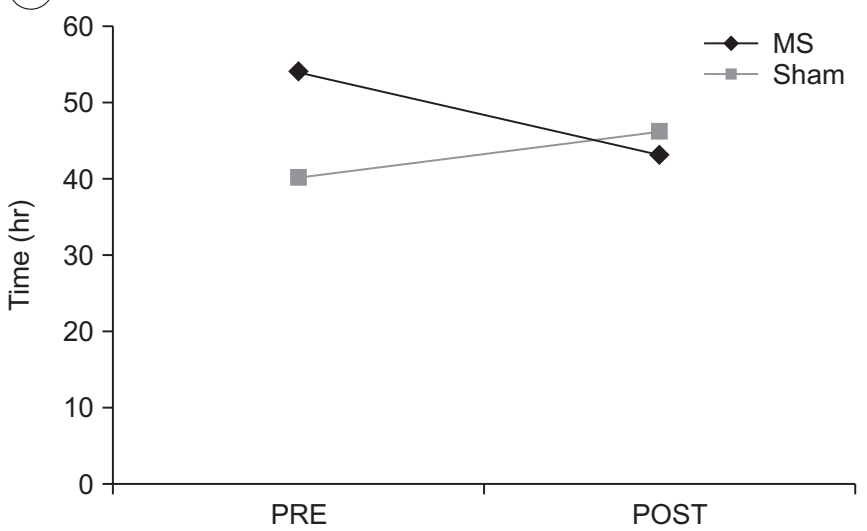

(C)

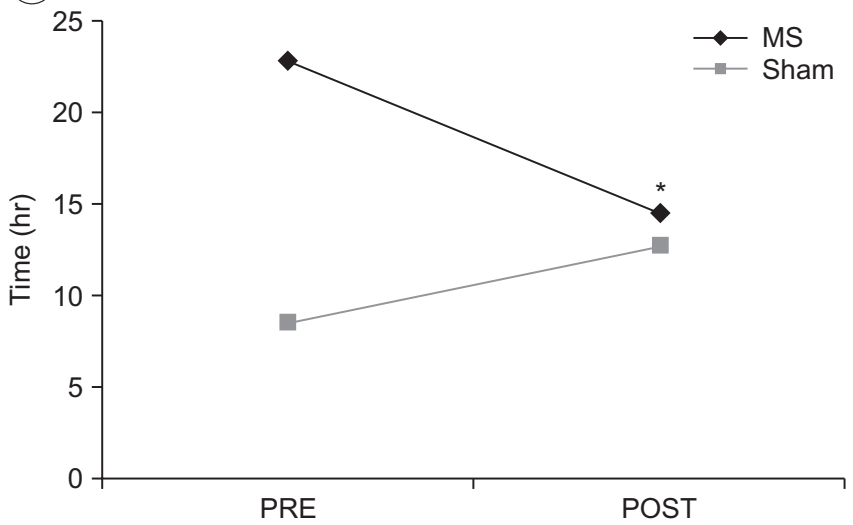

\section{Changes in frequency of defecation (times/week)}

The frequency of defecation increased from $1.7 \pm 0.7$ to $3.2 \pm 0.4$ weekly in the MS group and from $2.5 \pm 0.8$ to $3.1 \pm 1.0$ weekly in the Sham group, which was a significant increase in the MS group compared to the Sham group ( $\mathrm{p}=0.012$, Welch test). The number of patients with increased frequency of defecation per week after treatment was 12 in the MS group and 5 in the Sham group. The proportion of patients with increased defecation was significantly higher in the MS group $(p<0.01$, Fisher exact test).

\section{Changes in the BBS}

BBS is classified according to the shapes and hardness of the stool. Types 1 and 2 indicate hard stool whereas Types 3 and 4 indicate normal stool consistency. The types increased from $2.3 \pm 0.7$ (before treatment) to $3.5 \pm 0.5$ (after treatment) in the MS group, and from 2.6 \pm 0.8 to
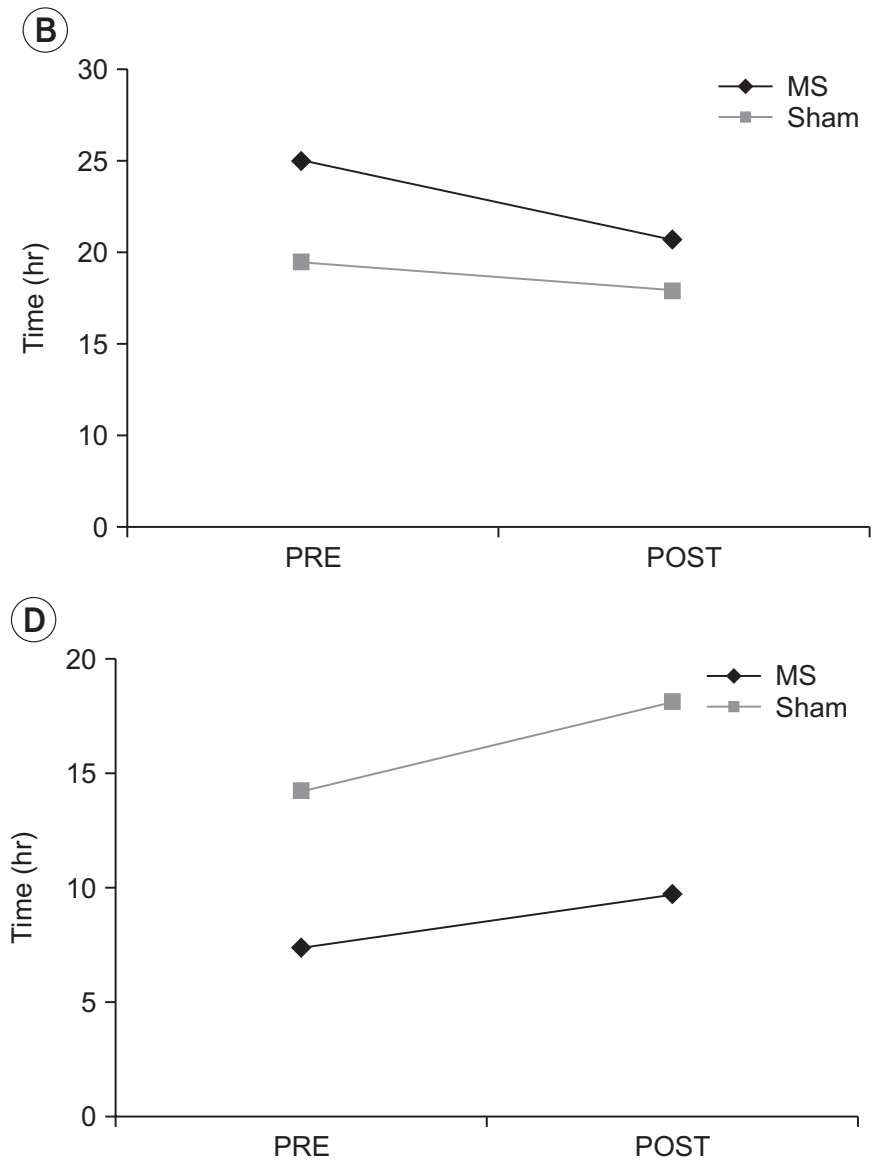

Fig. 3. Comparison of colon transit time (CTT) between transabdominal functional magnetic stimulation group (MS) and sham stimulation group (Sham): (A) total CTT, (B) right colon CTT, (C) left colon CTT, and (D) rectosigmoid CTT. PRE, pre-treatment; POST, post-treatment. ${ }^{*} \mathrm{p}<0.05$ by Student $\mathrm{t}$-test. 
$3.1 \pm 0.5$ in the Sham group, respectively. The proportion of patients with an increase in stool types was significantly higher in the MS group compared to the Sham group (10 in the MS group) ( $\mathrm{p}=0.013$, chi-square test).

\section{Changes in $\mathrm{K}-\mathrm{MBI}$}

K-MBI, which conveys that higher the score higher the degree of self-reliance, as a measure of patients' activities of daily living, was $36.9 \pm 31.4$ (before treatment) and $41.8 \pm 32.0$ (after treatment) in the MS group, and 27.8 \pm 22.0 (before treatment) and $35.3 \pm 30.0$ (after treatment) in the Sham group. There was no significant difference in the score between the two groups.

\section{Side effects due to FMS}

None of the participants showed any adverse reactions or any other complications during or after treatment with A-FMS.

\section{DISCUSSION}

We evaluated the effects of A-FMS on constipation (decrease in large intestine movements) in patients with brain injuries and investigated the effect of CTT, defecation frequency, and stool hardness. Statistically significant changes were observed with regards to several variables. The large intestine is a rather complex organ with autonomic, enteric and somatic nervous input. The vagus nerve (10th cranial nerve, CN X) and pelvic nerve (S2-4) are under the parasympathetic control wherein the vagus nerve innervates the ascending and transverse colons while the pelvic nerve innervates the descending colon. Auerbach's and Meissner's plexuses distributed within the enteric system and colonic wall promote peristaltic movement while mixing and advancing the stool in association with the harmonious movement of the bowel. When the stool reaches the rectum, rectal stretching causes simultaneous relaxation of puborectalis muscle and external anal sphincter. Concurrently, the abdominal muscles contract to increase the pressure in the abdomen to promote defecation [12].

FMS was introduced in 1994 to functionally stimulate the peripheral nerves [17]. Changes in the magnetic field induce an electric field and appropriate management of intensity and duration of the electric field generates current sufficient to excite the nerves [18]. Several recent studies have reported improvements in bowel function by stimulating the nerves with magnetic stimulation. Chiu et al. [19] reported a study about the effect of FMS in 16 patients with Parkinson disease during the first 10 minutes for the T9 spinous process for thoracic nerve stimulation and for the remaining 10 minutes for the lumbosacral nerve stimulation in the L3 spinous process for $20 \mathrm{~min}$ utes twice daily for 3 weeks with a significant decrease in CTT after application. Wang and Tsai [20] reported improvement in bowel function in 19 patients with intractable constipation aged 65 years or older by magnetic stimulation therapy for 3 weeks by following the protocol described by Chiu et al. [19]. Lin et al. [12] reported that FMS at the aforementioned sites among 13 patients with spinal cord injuries and 2 normal subjects increased rectal pressure and decreased CTT following application of FMS to the abdominal area at $10 \mathrm{~cm}$ above the symphysis pubis; they observed a higher rectal pressure compared to the pressure at the time of spinal stimulation and direct smooth muscle contraction as a result of pelvic nerve stimulation. Furthermore, Yoon et al. [13] showed a decrease in CTT, softened stool consistency and increased the frequency of defecation in 12 stroke patients with chronic constipation via transabdominal approach to stimulate pelvic nerve and local enteric system as well as the abdominal muscles directly in a relaxed supine position of these patients. In the present study, we applied the A-FMS through the abdomen while following the protocol described by Yoon et al. [13]. Depending on the frequency of the stimulation, the muscles may repeat the contraction and relaxation in low-frequency setting and maintain a constant contraction state in high-frequency setting. The stimulation frequency of $40 \mathrm{~Hz}$ maintains the constant contraction state [21], which leads to physiological defecation activities such as the Valsalva maneuver. A sufficient resting period of 6 seconds after 3 seconds of stimulation was provided to prevent fatigue of the abdominal muscles during 20 minutes of treatment.

The increase in BSS (indicating decreased stool hardness) and the frequency of defecation are the results of complex stimulation of muscles and nerves caused by AFMS. However, there was no statistically significant difference between the two groups with respect to change in total CTT and the change in segmental CTT except in the segmental CTT in the left colon. Various methods for setting more specific and effective treatment guidelines 
are necessitated. Herein, in our study we did not evaluate patient's body mass index or the anteroposterior (AP) diameter of the abdomen-distance from the stimulator to the target structure should be different in each patientthereby rendering a limitation of the current study.

In conclusion, the present study demonstrated that the use of A-FMS in patients with constipation after brain injury resulted in decreased but equivocal in CTT, increased frequency of defecation and decreased stool hardness. It is proposed that A-FMS should be made available as an additional therapeutic option for the treatment of constipation in brain-injured patients.

\section{CONFLICT OF INTEREST}

No potential conflict of interest relevant to this article was reported.

\section{REFERENCES}

1. Stewart WF, Liberman JN, Sandler RS, Woods MS, Stemhagen A, Chee E, et al. Epidemiology of constipation (EPOC) study in the United States: relation of clinical subtypes to sociodemographic features. Am J Gastroenterol 1999;94:3530-40.

2. Pare P, Ferrazzi S, Thompson WG, Irvine EJ, Rance L. An epidemiological survey of constipation in Canada: definitions, rates, demographics, and predictors of health care seeking. Am J Gastroenterol 2001;96:31307.

3. Hollerbach S, Kamath MV, Lock G, Scholmerich J, Upton AR, Tougas G. Assessment of afferent gut: brain function using cerebral evoked responses to esophageal stimulation. Z Gastroenterol 1998;36:313-24.

4. Karakula-Juchnowicz H, Dzikowski M, Pelczarska A, Dzikowska I, Juchnowicz D. The brain-gut axis dysfunctions and hypersensitivity to food antigens in the etiopathogenesis of schizophrenia. Psychiatr Pol 2016; 50:747-60.

5. Li J, Yuan M, Liu Y, Zhao Y, Wang J, Guo W. Incidence of constipation in stroke patients: a systematic review and meta-analysis. Medicine (Baltimore) 2017;96: e7225.

6. Lin CJ, Hung JW, Cho CY, Tseng CY, Chen HY, Lin FC, et al. Poststroke constipation in the rehabilitation ward: incidence, clinical course and associated fac- tors. Singapore Med J 2013;54:624-9.

7. Camara-Lemarroy CR, Ibarra-Yruegas BE, GongoraRivera F. Gastrointestinal complications after ischemic stroke. J Neurol Sci 2014;346:20-5.

8. Lin VM, Frost FS, Lee SB, Cadwell J. High-frequency magnetic stimulation of the bowel in man: a pilot study [Abstract]. Muscle Nerve 1994;17:1098.

9. Dinning PG, Fuentealba SE, Kennedy ML, Lubowski DZ, Cook IJ. Sacral nerve stimulation induces pancolonic propagating pressure waves and increases defecation frequency in patients with slow-transit constipation. Colorectal Dis 2007;9:123-32.

10. Govaert B, Maeda Y, Alberga J, Buntzen S, Laurberg S, Baeten CG. Medium-term outcome of sacral nerve modulation for constipation. Dis Colon Rectum 2012; 55:26-31.

11. Lee KJ, Kim JH, Cho SW. Short-term effects of magnetic sacral dermatome stimulation for idiopathic slow transit constipation: sham-controlled, cross-over pilot study. J Gastroenterol Hepatol 2006;21(1 Pt 1):47-53.

12. Lin VW, Nino-Murcia M, Frost F, Wolfe V, Hsiao I, Perkash I. Functional magnetic stimulation of the colon in persons with spinal cord injury. Arch Phys Med Rehabil 2001;82:167-73.

13. Yoon YS, Kim ES, Choi JH, Jeon JH, Lee MY, Lee KJ. The effect of functional magnetic stimulation in stroke patients with chronic constipation. Gazz Med Ital 2016;175:421-6.

14. Drossman DA. The functional gastrointestinal disorders and the Rome III process. Gastroenterology 2006; 130:1377-90.

15. Lim YH, Kim DH, Lee MY, Joo MC. Bowel dysfunction and colon transit time in brain-injured patients. Ann Rehabil Med 2012;36:371-8.

16. Robinson LR. Magnetic stimulation of the central and peripheral nervous systems. In: Dumitru D, Amato AA, Zwarts M, editors. Electrodiagnostic medicine. 2nd ed. Philadelphia: Henley \& Belfus; 2002. p. 41527.

17. Davey K, Luo L, Ross DA. Toward functional magnetic stimulation (FMS) theory and experiment. IEEE Trans Biomed Eng 1994;41:1024-30.

18. Lin VW, Hsieh C, Hsiao IN, Canfield J. Functional magnetic stimulation of expiratory muscles: a noninvasive and new method for restoring cough. J Appl Physiol (1985) 1998;84:1144-50. 
19. Chiu CM, Wang CP, Sung WH, Huang SF, Chiang SC, Tsai PY. Functional magnetic stimulation in constipation associated with Parkinson's disease. J Rehabil Med 2009;41:1085-9.

20. Wang CP, Tsai PY. Efficacy of spinal magnetic stimulation in elderly persons with chronic constipation. J
Chin Med Assoc 2012;75:127-31.

21. Sohn MK, Cho KH, Yoon SJ, Lee TS, Lee KJ. Effects of functional magnetic stimulation on hemiplegic shoulder subluxation. J Korean Acad Rehabil Med 2007;31: 48-55. 